\title{
WCDRR AND THE CEOS ACTIVITIES ON DISASTERS
}

\author{
Ivan Petiteville $^{\mathrm{a}}$, Chu Ishida ${ }^{\mathrm{b}}$, Jens Danzeglocke ${ }^{\mathrm{c}}$, Andrew Eddy ${ }^{\mathrm{d}}$, Francesco Gaetani ${ }^{\mathrm{e}}$, Stuart Frye ${ }^{\mathrm{f}}$, Bob Kuligowski ${ }^{\mathrm{g}}$, Simona \\ Zoffoli $^{\text {h }}$, Michael Poland ${ }^{\mathrm{i}}$, Brenda Jones ${ }^{\mathrm{j}}$ \\ ${ }^{\text {a }}$ European Space Agency (ESA), ESRIN, Via G.Galilei, CP 64, 00044 Frascati, Italy - Ivan.Petiteville@esa.int \\ b Japan Aerospace Exploration Agency (JAXA), Tsukuba Space Center 2-1-1 Sengen, Tsukuba, Ibaraki, 305-8505 Japan - \\ ishida.chu@jaxa.jp \\ ${ }^{c}$ German Aerospace Center (DLR), Space Administration, Earth Observation, Bonn, Germany - jens.danzeglocke@dlr.de \\ ${ }^{\mathrm{d}}$ Athena Global, La Frouste, 04150 Simiane-la-Rotonde, France - andrew.eddy@ athenaglobal.com \\ ${ }^{\mathrm{e}}$ Group on Earth Observations (GEO) Secretariat, 7 bis, avenue de la Paix, Case postale 2300, CH-1211 Geneva 2, Switzerland- \\ fgaetani@geosec.org \\ ${ }^{\mathrm{f}}$ National Aeronautics and Space Administration (NASA), Code 474, NASA Goddard Space Flight Center, 8800 Greenbelt Road, \\ Greenbelt Maryland 20771, United States - stuart.frye@nasa.gov \\ ${ }^{g}$ National Oceanic and Atmospheric Administration (NOAA), NCWCP E/RA2, 5830 University Research Court, 2nd Floor, Office \\ \#2828, College Park, MD 20740 USA - Bob.Kuligowski@ noaa.gov \\ ${ }^{\mathrm{h}}$ Agenzia Spaziale Italiana (ASI), Osservazione della Terra, Via del Politecnico - 00133 - Rome - Italy - simona.zoffoli@asi.it ${ }^{\mathrm{i}}$ U.S. \\ Geological Survey (USGS), Hawaiian Volcano Observatory, 51 Crater Rim Drive \\ Hawaii National Park, HI 96718, United States - mpoland@usgs.gov \\ ${ }^{\mathrm{j}}$ U.S. Geological Survey (USGS), USGS EROS Center, 47914 252nd St, Sioux Falls, SD 57198, United States - \\ bkjones@usgs.gov
}

\section{THEME: Disasters}

KEY WORDS: satellite, international cooperation, disaster risk reduction

\begin{abstract}
:
Agencies from CEOS (Committee on Earth Observation Satellites) have traditionally focused their efforts on the response phase. Rapid urbanization and increased severity of weather events has led to growing economic and human losses from disasters, requiring international organisations to act now in all disaster risk management (DRM) phases, especially through improved disaster risk reduction policies and programmes. As part of this effort, CEOS agencies have initiated a series of actions aimed at fostering the use of Earth observation (EO) data to support disaster risk reduction and at raising the awareness of policy and decision-makers and major stakeholders of the benefits of using satellite EO in all phases of DRM.
\end{abstract}

CEOS is developing a long-term vision for sustainable application of satellite EO to all phases of DRM. CEOS is collaborating with regional representatives of the DRM user community, on a multi-hazard project involving three thematic pilots (floods, seismic hazards and volcanoes) and a Recovery Observatory that supports resilient recovery from one major disaster. These pilot activities are meant as trail blazers that demonstrate the potential offered by satellite EO for comprehensive DRM.

In the framework of the $20153^{\text {rd }}$ World Conference on Disaster Risk Reduction (WCDRR), the CEOS space agencies intend to partner with major stakeholders, including UN organizations, the Group on Earth Observations (GEO), international relief agencies, leading development banks, and leading regional DRM organisations, to define and implement a 15-year plan of actions (20152030) that responds to high-level Post-2015 Framework for Disaster Risk Reduction priorities. This plan of action will take into account lessons learned from the CEOS pilot activities.

\section{INTRODUCTION}

Over the last decade, efforts from the major space agencies in the world have mostly focused on the response phase of disasters management leading for instance to the establishment of successful operational services such as the International Charter 'Space and Major Disasters'. Rapid urbanization and increased severity of weather events has led to growing economic and human losses from disasters, every year. International organisations have addressed this issue through improved disaster risk reduction (DRR) policies and programs. Following the same trend, space agencies are now addressing the challenges presented by the diversity and scale of satellite observations being applied to global disaster prevention, mitigation and preparedness while maintaining their current efforts on emergency-related activities.

Large-scale use of satellite Earth observation (EO) for DRM is hindered by a lack of user awareness of possible applications and the complexity of acquiring data from a wide range of providers. Satellite EO plays a complementary role to other data sources, but offers unique scope and coverage. As in-situ information can be difficult to obtain or is simply unavailable, remote sensing data may be the only reliable data source in some areas, and can be downscaled to local needs if the sensor's resolution allows it.

As an objective and global data source, remote sensing from space offers a first layer of information for global monitoring of 
the evolution of risk, to support implementation of post-2015 framework progress. It can provide both regularly updated hazard information and exposure data. Risk managers also require DRM tools specifically adapted to user needs that integrate data and generate risk information products.

Through the CEOS, space agencies are seeking to provide coordinated satellite observations in support of risk assessment and in particular to disaster risk reduction. CEOS is developing a long-term vision for sustainable application of satellite EO to all phases of DRM that would require the active participation of practitioners capable of transforming satellite data into risk information products that can be directly used by national and local decision makers. It anticipates multi-hazard contributions that are global in scope, but defined through cooperative action at the regional level.

\section{CURRENT INVOLVEMENT OF SPACE AGENCIES IN DISASTERS AND NEW PERSPECTIVES AFTER WCDRR.}

\subsection{Space agencies activities related to disasters}

Space agencies' operational capabilities are mostly focused on post-disaster monitoring and damage assessment. Early warning is still a research issue. Nevertheless, there are several on-going research and development projects that demonstrate the benefits of EO data use during all phases of disaster management. For instance in the Group on Earth Observations (GEO) framework, space agencies coordinate their resources and efforts to facilitate access to space information products and services to support disaster management decision-making. These efforts have resulted in several global and regional programs of international co-operation. Space agencies are used to cooperating at an international level within the CEOS framework with non-space communities. Some examples of cooperation activities within CEOS are provided in section 3 "CEOS DRM Pilots and the Recovery Observatory". Only these coordinated efforts between space agencies have made possible some international initiatives that could not have been fully supported by a single space data provider.

Among several examples of successful international cooperation involving space agencies in the disasters and climate change fields, we could mention:

- the International Charter 'Space and Major Disasters', that aims at providing a unified system of space data acquisition and delivery to those affected by natural or man-made disasters, helping to mitigate the effects of disasters on human life and property;

- Sentinel-Asia, that supports disaster management activities in the Asia-Pacific region by applying space based technology and web-GIS technology, and working on all phases of disaster, preparedness, response and recovery;

- the GEO Global Forest Observations Initiative (GFOI) that fosters the sustained availability of observations for national forest monitoring systems as requested by the United Nations Framework Convention on Climate Change (UNFCCC);

- the UNFCCC and the Intergovernmental Panel on Climate Change (IPCC) with the provision to the climate research community of Essential Climate Variables (ECVs) that are important to a full understanding of the climate system; - the Geohazard Supersites and Natural Laboratories (GSNL), through which CEOS provides large volumes of satellite data to support scientific research into fault zones and volcanoes at designated sites, in partnership with local and international scientists using in-situ data.

Many of these initiatives are the subject of current on-going research. The contribution from space agencies to climate change is also very relevant to disaster management as damages from extreme weather-related hazards may triple by 2100 .

Satellite EO data use for DRM is mostly complementary to other sources of data, but where in-situ information is limited or where other forms of monitoring are affected by the hazard, EO data may be the only source of information available. Only satellite EO offers the consistent coverage and scope to provide a synoptic overview of large areas, repeated regularly. Satellite EO can be used to compare risk across different countries, day and night, in all weather conditions, and in transboundary areas where information might be difficult to collect. In addition, satellite data offers a unique means for monitoring the progress of the post-2015 framework by offering a synoptic view of the evolution of risk on a global basis, using globally comparable metrics. In the GEO framework, space agencies are working with in-situ data and information providers to create new information products better targeted to the user needs understandable also to non-specialists. Several examples of concrete use by end users and decision makers of information derived from remote sensing data can be found in the CEOS brochure "Satellite Earth Observation in Support of Disaster Risk Reduction" edited by the European Space Agency (ESA) at the occasion of the $3^{\text {rd }} \mathrm{UN}$ World Conference on Disaster Reduction and Recovery (WCDRR) held in Sendai (Japan) from 14 to 18 March, 2015.

\subsection{WCDRR}

The $3^{\text {rd }}$ United Nations World Conference on Disaster Risk Reduction (WCDRR) took place in Sendai (Japan) on 14-18 March 2015. The previous edition of the WCDRR was held in Kobe (Japan), in 2005. The 2015 WCDRR event was attended by some 20 Heads of States and Governments, and 135 Ministers and Vice-Ministers; the conference hosted 8000 participants.

The WCDRR marks a major milestone in disaster management as the common focus between actors is now disaster risk reduction. In his opening speech, the UN Secretary General recalled that "six billion dollars allocated each year can result in savings of up to \$US 360 billion by 2030", asking for efforts to be broadened to all phases of disaster management and not solely on response. In echo to this call, during the opening ceremony, Japanese Prime Minister Abe announced \$US 4 billion to support implementation of the "Sendai Cooperation Initiative for Disaster Risk Reduction" over the next four years. This future initiative was further detailed in the front page of Japan's largest newspaper; it includes an Early-Warning-System (EWS) providing necessary observations, predictions and warning means using satellite and information on infrastructure. With the renegotiation of the post-2015 framework on disaster risk reduction, several national Delegates asked for sustainable solutions to be rapidly put in place. Several talks explicitly advocated for the use of satellites, which shows recent progress made in the increasing awareness of decision makers of the usefulness of satellite EO for disaster risk reduction. Recurring disaster themes of concern included floods, earthquakes, volcanoes and landslides, which are closely related to CEOS disaster activities. 
During the WCDRR, several politicians' statements contributed to a general sentiment that all resources must be used or better used, including satellite technology, provided that the complexity of the technology is hidden from the end user. Several speakers also highlighted the "last mile" issue: in several cases, adequate and useful risk information exists but does not reach national or the local decision makers. Now there is strong political support for using all means available, there is a new and unique opportunity for space data providers to foster the use of remote sensing data through a new partnership with relevant stakeholders.

\section{CEOS DRM PILOTS AND THE RECOVERY OBSERVATORY}

Through its Working Group on Disasters, CEOS Agencies have elaborated a strategy aiming at fostering the use of satellite EO in disaster management. A series of activities were initiated two years ago to demonstrate the validity of various concepts that form that strategy. These pilots were approved for implementation in April 2014 and will run to the end of 2017.

CEOS member agencies are developing three single-hazard thematic pilots, in collaboration with regional representatives of the DRM user community, focusing on floods, volcanoes, and seismic hazards. One of the main objectives of these Pilots is to demonstrate that close cooperation between space agencies, and close dialogue with the user community, can bring a significant added value to DRM by better responding to the users' needs in terms of data suitability, quality, timeliness, and access. It also serves as a good platform to exercise international cooperation with key stakeholders and practitioners. Besides it allows space agencies to define and regularly improve a set of processes and methods aiming at ensure a proper provision of satellite EO data by space agencies and a proper use of this data and derived information by intermediate and end users. In addition to the three pilots, the CEOS Working Group on Disasters is developing a concept called the Recovery Observatory with the main objective of helping reconstruction by collecting and distributing all types of free data (e.g. satellite, in-situ, socioeconomic, model outputs) for a multi-year period, and encouraging the development of new satellite-based products to support early recovery planning and recovery implementation monitoring.

\subsection{Flood Pilot}

The main goal of that pilot is to demonstrate the effective application of satellite EO to the full cycle of flood management at global and regional/local scales by:

- Integrating existing near-real time global flood monitoring and modelling systems.

- $\quad$ Linking global systems to regional end-to-end pilots that produce high-resolution flood mitigation, warning and response products and deliver flood and flash flood related services in:

- The Caribbean (with particular focus on Haiti);

- Southern Africa, including Namibia, South Africa, Zambia, Zimbabwe, Mozambique and Malawi;

- Southeast Asia (with particular focus on the lower Mekong Basin and Western Java, Indonesia).
- Developing new end products and services to better deliver flood related information and to validate satellite EO data and products with end users, including retrospective products working from archived EO flood extent data.

- Encouraging regional in-country capacity building to access EO data and integrate into operational systems and flood management practices.

Benefits of CEOS activity on floods: there are a large number of flood related initiatives underway around the world, in every region of the world, and on a global basis, working with different data sets and sensors and developing different products. While the advent of rapid mapping using satellite EO has led to some degree of standardization in the context of EO based response capabilities (such as the International Charter Space \& Major Disasters, Sentinel-Asia, etc.), there is a need for greater cooperation across existing projects to develop common approaches on how to collect data for flood monitoring and the prevention of flood damage, and how to exploit data and derived information from them. Furthermore, there is a strong need for comparison of results across local, national and global efforts to enable global systems to become more accurate and to chart a path for sustained local and national monitoring on an on-going basis across the world. The regional Pilots cover the Caribbean, southern Africa, and parts of Southeast Asia, and are leveraging multiple ongoing projects in these regions by using "Flood Dashboards" to consolidate available flood-related data in a single location in a manner that is easy to use and interpret. These regional Flood Dashboards include satellite-derived rainfall and flood extent, model forecasts of rainfall, and hydrologic model forecasts, along with available in situ data. The ability to overlay multiple fields on map backgrounds makes it easy to interpret the data and compare related data from different sources. The regional Pilots are also working to leverage training efforts in the involved projects to make users aware of the Flood Dashboards and also to solicit feedback to increase their usefulness. Meanwhile, the global Pilot is coordinating information from several different global flood monitoring systems and also providing a platform for validating these global systems using data from the regional Pilots. All of these Pilots also provide a common platform for requesting and sharing data from space agencies that do not routinely make data available free of cost to end users, enabling users in under-resourced countries to benefit from these data.

CEOS offers the breadth of membership and sensor platforms to provide the full range of necessary data to support an effort that brings together end-users, practitioners, and satellite operators. CEOS offers a unique venue to improve the efficiency of satellite observations through coordination of observing strategies across multiple agencies and comparison of products to improve the standardization of observing practices in support of flood monitoring products. The pilot will generate new products as a result of the combination of several satellite sources, which enables a higher degree of accuracy. In some areas, the use of more than one mission with similar instrument type over a specific geographical zone can be harmonized to eliminate redundant data collects and allow new areas to be imaged. 


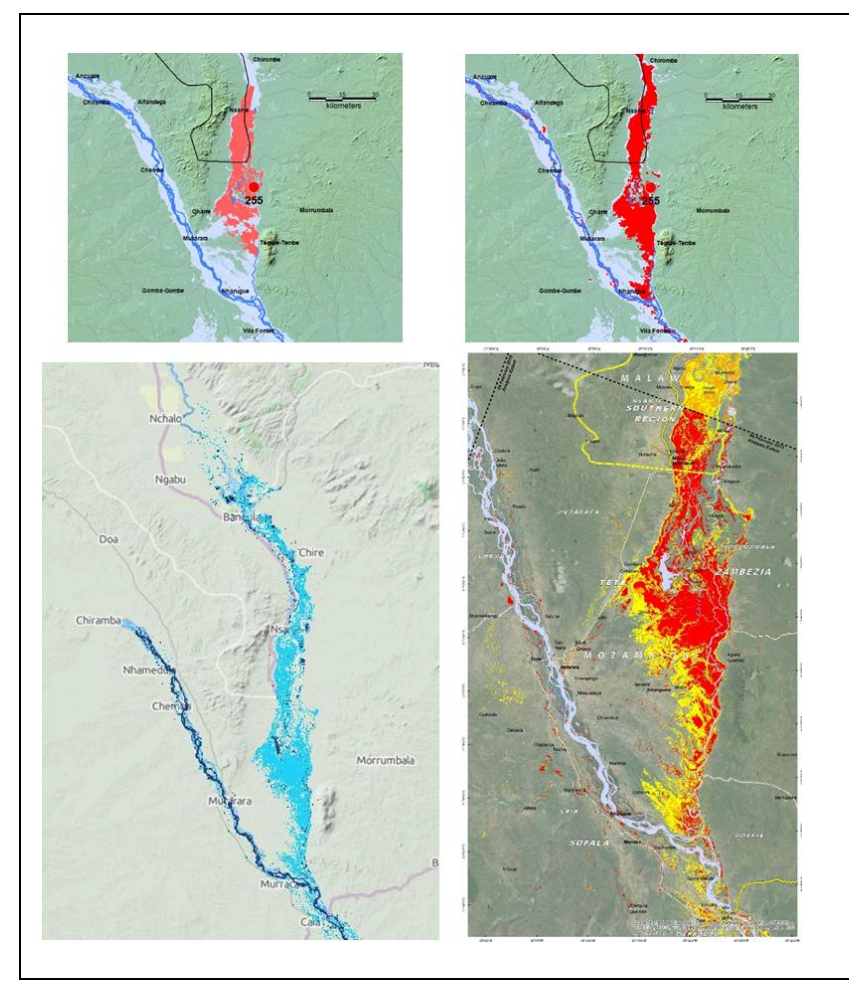

Figure 1. Malawi 2015 flood extent maps, courtesy of CIMA Foundation and UNOSAT.

Figure 1 shows a series of flood maps generated early 2015 using several satellite EO optical and radar sensors in order to perform cross-validation of products: MODIS on Jan. 26 (top right), EO-1 on Jan. 26 (top left), Sentinel-1 on Jan. 22 (bottom left) and Radarsat-2 on Jan. 21, Jan. 30, Feb. 4 (bottom right). This cross-validation completed by in-situ validation (as depicted in fig. 2) is necessary in order to improve the accuracy of the predictions of the flood extent. Each day predictions are gathered by a variety of computer models run on ground and satellite readings and made available to end users. The Flood Pilot team works with regional users in Southern Africa, Caribbean/Central America, and Southeast Asia to integrate satellite monitoring and modeling with local knowledge.

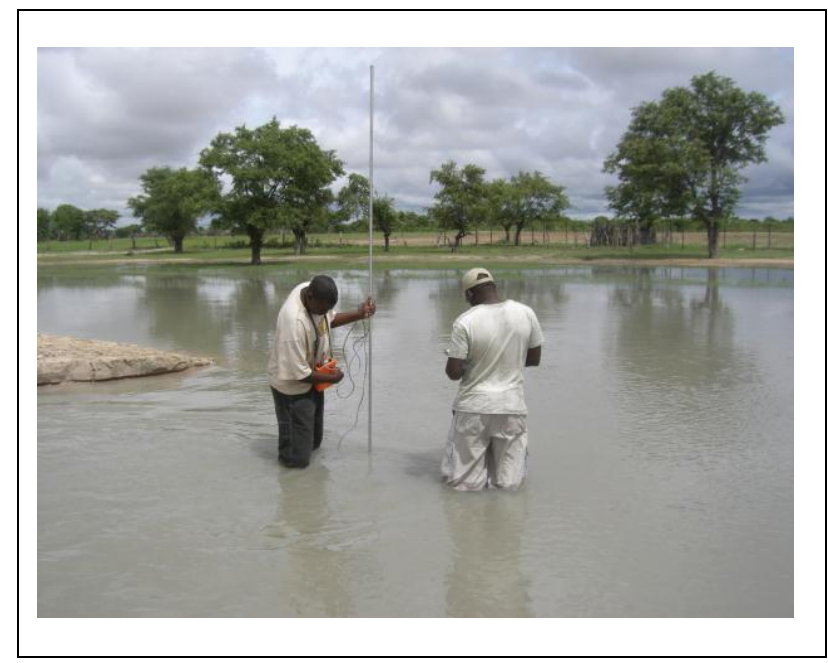

Figure 2. Hydrologists in Namibia validating flood water depth and river flow rates against satellite data, courtesy of NASA

\subsection{Volcano Pilot}

The volcanoes pilot exploits the instruments of several EO satellite missions to achieve the following objectives:

- Demonstrating comprehensive monitoring of Holocene era volcanoes in the Latin American volcanic arc, as a precursor to global monitoring of all Holocen era volcanoes.

- Developing new protocols and products over active volcanoes where EO data collects are already taking place through the Geohazard Supersites initiative (GSNL Hawaii, Iceland, and Italy).

- Demonstrating operational monitoring over a large scale eruption (e.g. Merapi 2010) during 2014-2017.

Benefits of CEOS activity on volcanoes: this pilot represents a stepping-stone towards the long-term goals of the Santorini Report with respect to volcanic activity, namely: 1) global background observations at all Holocene volcanoes; 2) weekly observations at restless volcanoes; 3) daily observations at erupting volcanoes; 4) development of novel measurements; 5) 20-year sustainability; and 6) capacity-building. By completing a regional study of volcanic activity in Latin America (sustained regional monitoring over time), the CEOS pilot will demonstrate the feasibility of a global monitoring programme over all Holocene volcanoes. The development of a comprehensive monitoring programme that includes all potentially active volcanoes and works in close cooperation with local and national end-users will serve as a showcase to demonstrate the value of EO-based monitoring, especially in areas where volcano observatories have fewer resources. Such an ambitious monitoring programme is only possible through a strong coordination between in situ and space Agencies and the collective effort of many agencies working together under an umbrella such as CEOS to achieve a mutually sought-after goal. In-depth monitoring using EO at established volcanic hotspots (the GSNL supersites) will enable the improvement of operational protocols for monitoring of active volcanoes. Data contributions from different agencies enable a truly multiplatform approach to be developed. These protocols and new products can be demonstrated in the event of a major eruption during the lifetime of the pilot.

Figure 3 provides an example of concrete use of satellite EO in early warning. Interferometry techniques have been applied to radar images to detect a volcano deformation that has so far been primarily associated with a larger (M5.6w) earthquake on October $22^{\text {nd }}, 2014$. This earthquake resulted in maximum displacements of $15 \mathrm{~cm}$ towards the satellite in a region $\mathrm{SW}$ of Chiles volcano.

The majority of deformation can be explained by oblique slip on a fault at depths $\sim 1.5-3 \mathrm{~km}$ dipping WNW. The orientation of this fault aligns well with faults mapped from regional topographic structures. Neither Chiles or Cerro Negro have erupted for a long time but above results associated to a remote monitoring by the USGS of thousands of small earthquakes, led scientists to believe that this could produce a very large eruption. This was judged sufficiently alarming by the Civil Defense in Colombia that they ordered the evacuation of 12,000 people. 


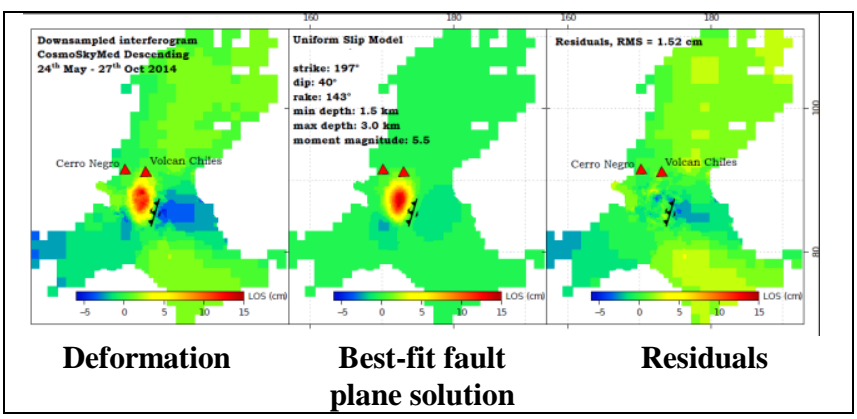

Figure 3. Ground deformations of both Chiles and Cerro Negro de Mayasquer volcanoes (Ecuador-Columbia border), courtesy of Instituto Geophysico, Ecuador.

\subsection{Seismic Hazards Pilot}

This pilot is characterized by three main objectives:

- Supporting the generation of globally self-consistent strain rate estimates and the mapping of active faults at the global scale by providing EO InSAR and optical data and processing capacities to existing initiatives, such as the iGSRM [Wide extent satellite observations].

- Supporting and continuing GEO's Geohazard Supersites and Natural Laboratories (GSNL) initiative for seismic hazards and volcanoes [Satellite observations focused on supersites].

- Developing and demonstrating advanced science products for rapid earthquake response [>Magnitude 5.8].

Benefits of CEOS activity on seismic hazards: while the CEOS activity builds on some major, existing initiatives, the involvement of CEOS offers clear added value. For wide area observations, CEOS offers a coordinated approach to observations, eliminating overlap and duplication, allowing the gathering of data necessary for large-scale ground motion and fault mapping. These high-resolution, continuous coverage, strain rate and fault maps will in turn become an essential input to probabilistic seismic hazard estimation, today based only on seismicity models and historical earthquake catalogues (as in the Global Earthquake Model project); CEOS also offers a framework in which users and practitioners can be federated; finally CEOS offers new tools developed through contributions of CEOS pilot partners to enable the generation of advanced products, through the development of an exploitation platform. The involvement of a range of CEOS agencies allows more satellites to be used to collect data for rapid product generation, and allows satellite agencies to work together to establish standard collection protocols to support the generation of such products in the aftermath of earthquakes. The pilot aims at demonstrating on a regional scale how a global EO-based strain model would improve strain measurements for a better understanding of seismic hazards, developing the existing GEO Geohazard Supersites and Natural Laboratories and showcasing EO-based science products for rapid response to earthquake events.

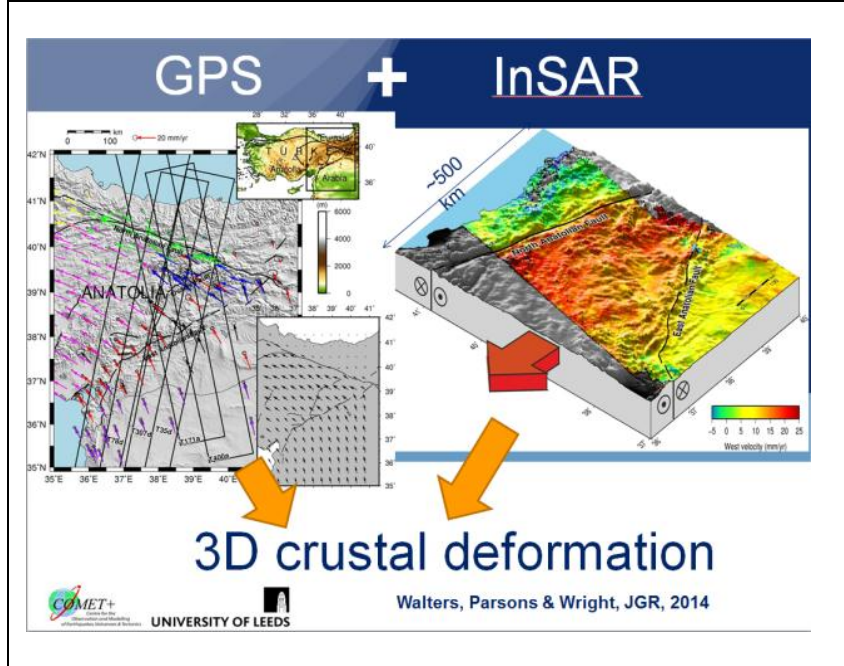

Figure 4. 3D crustal deformation in the regions of the North and East Anatolian faults, courtesy of COMET+.

The combination of in-situ GPS measurements and interferometry SAR data allows the visualization of crustal deformation in $3 \mathrm{D}$, as illustrated in figure 4.

\subsection{Recovery Observatory}

In addition to the three thematic pilots, CEOS Agencies are setting up a Recovery Observatory (RO) that will showcase how agencies can improve collaboration in the aftermath of a major disaster on the scale of Typhoon Haiyan or the Haiti Earthquake of 2010

As of January 1, 2015, the RO is ready for activation, awaiting a major event that will allow satellite agencies, international recovery stakeholders and value adding partners to showcase the possibilities offered to the recovery planning and monitoring community by standard use of satellite data.

UNOSAT, UNDP and GFDRR/World Bank have joined the RO Oversight Team (ROOT), which is in charge of recommending the RO triggering and managing the preparation, triggering, establishment and operations of the RO). Discussions are ongoing with the IFRC (Red Cross), and the ROOT hopes to engage UNEP in spring 2015. Working closely with international stakeholders, the ROOT has agreed to collaborate on a few rapid assessments and support to Post Disaster Needs Assessment in order to better prepare for the Recovery Observatory. The ROOT is refining the appreciation of the requirements with these stakeholders, and establishing a target list of possible applications for a few key disaster types. During the early recovery for Typhoon Pam over Vanuatu, the ROOT worked closely with the World Bank to determine how satellite assets could be brought to bear to support rapid assessment of damages to housing, transport infrastructure and the agricultural sector. A separate detailed paper is being presented o the Recovery Observatory at this conference (see Hosford et alii., The CEOS Recovery Observatory Pilot, ISRSE 36). 


\section{CREATING A PARTNERSHIP FOR THE APPLICATION OF SATELLITE EO TO THE POST-2015 FRAMEWORK FOR DISASTER REDUCTION}

As explained above, now that the post-2015 framework is endorsed by the international community, space agencies will prepare and implement a 15-year plan for the period 2015-2030 to address the priorities identified by the major stakeholders involved in disaster risk reduction and to fill the gaps wherever feasible with the resources available. All relevant actors will be consulted and involved in the generation of a plan that shall be ready within one year following the endorsement of the new framework. Organisations such as CEOS and its member space agencies seek to develop stronger relationships with international and regional coordinating agencies such as UN International Strategy for Disaster Reduction (UNISDR), the World Bank Global Framework for Disaster Risk Reduction (GFDRR), UN ESCAP, European Institutions, other leading international financial institutions and donor agencies, as well as relief agencies such as the IFRC (Red Cross). This partnership should help space agencies to understand what the highest priority needs are from the disaster risk management community including the regional and local levels, and to implement targeted solutions, in close cooperation with the relevant actors. Strong international cooperation is needed to implement robust mechanisms aimed at better sharing of information.

In addition to major stakeholders, user communities have an important role to play in the identification of priorities for space agency attention and must stay engaged throughout the implementation and operation of the resulting programmes. In order to maximize space agency contributions to the DRM Community, space agencies require that user needs be collated as a set that forms a coherent global strategy. A user-driven approach helps space agencies to better tailor their provision of data and products and to provide a useful feedback on the added value of remote sensing data. The "User communities" involve all the actors along the chain that goes from the data provider to the final end-users including intermediary users that transform the data into actionable information that can be directly used by the end-users, such as local, regional or national authorities. Furthermore, it is also important for end users to engage with one another and cooperate collectively at those levels to share information on how to access EO data and products for disaster risk reduction.

Following the successful model of the CEOS disaster-related pilots, a progressive implementation is being considered. Besides, given the current uncertainties related to the high-level needs of the user community and the limited resources that can be allocated initially by the various partners, a non-binding framework is being considered whereby partners will contribute on a voluntary basis with contributions in kind.

\section{CONCLUSION}

Following the encouraging results obtained by the CEOS Working Group on Disasters with the single and multi-hazard pilot activities and the interest from several DRM key stakeholders to join and several positive statements made by end users involved in those pilots, space agencies intend to partner with all motivated stakeholders to create a series of endto-end projects that address high priorities in the field of DRR, in line with the post-2015 framework for disaster risk reduction, just approved in Sendai.

\section{REFERENCES}

Petiteville, I., 2015, CEOS brochure "Satellite Earth Observation in Support of Disaster Risk Reduction - Special 2015 WCDRR", European Space Agency (ESA), Frascati, Italy, pp. 84.

Petiteville, I. (et alii.), "International Collaboration of Space Agencies to Support Disaster Preparedness and Response and Country Risk Management", long abstract for oral presentation, $5^{\text {th }}$ International Disaster Risk Conference, Davos, Switzerland, August 2014. 